\title{
BMJ Open Association between pregravid physical activity and family history of stroke and risk of stillbirth: population-based cohort study
}

\author{
Grace M Egeland, ${ }^{1}$ Grethe S Tell, ${ }^{1}$ Øyvind Næss, ${ }^{2}$ Jannicke Igland, ${ }^{1}$ Kari Klungsøyr ${ }^{1}$
}

To cite: Egeland GM, Tell GS, Næss $\emptyset$, et al. Association between pregravid physical activity and family history of stroke and risk of stillbirth: population-based cohort study. BMJ Open 2017;7:e017034. doi:10.1136/ bmjopen-2017-017034

- Prepublication history for this paper is available online. To view these files please visit the journal online (http://dx.doi. org/10.1136/bmjopen-2017017034).

Received 3 April 2017

Revised 12 June 2017

Accepted 28 June 2017

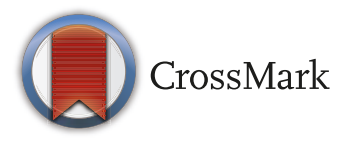

1Department of Global Public Health and Primary Care, Norwegian Institute of Public Health, \& Professor, University of Bergen, Bergen, Norway

${ }^{2}$ Institute of Health and Society, University of Oslo, Oslo, Norway

Correspondence to

Dr Grace M Egeland;

G.Egeland@uib.no

\section{ABSTRACT}

Objectives To evaluate whether family history of disease and pregravid lifestyle and cardiovascular risk factors are associated with subsequent stillbirth delivery.

Design Prepregnancy cohort study.

Setting Cohort Norway regional health surveys (19942003) linked to Medical Birth Registry of Norway for deliveries through 2012.

Participants 13497 singleton births $(\geq 22$ weeks gestation) in 8478 women.

Main outcome measure Risk of stillbirth evaluated by Poisson regression.

Results Mean (SD) length of follow-up was 5.5 (3.5) years. In analyses adjusting for baseline age and length of follow-up, $\geq 3$ hours of baseline past-year vigorous physical activity per week (resulting in shortness of breath/sweating) was associated with increased risk of stillbirth compared with $<1$ hour/week of vigorous activity (incidence rate ratio, IRR $2.46 ; 95 \% \mathrm{Cl} 1.23$ to 4.90 ). In contrast, baseline past-year light physical activity of $\geq 3$ hours per week associated with reduced risk of stillbirth compared with $<3$ hours of light physical activity per week (IRR $0.53 ; 95 \% \mathrm{Cl} 0.30$ to 0.93 ). A family history of stroke associated with increased risk of stillbirth delivery (IRR 2.53; $95 \% \mathrm{Cl} 1.06$ to 6.01 ). Because overweight/obese women may experience shortness of breath and sweating with less physical exertion than normal weight women, a sensitivity analysis was conducted limited to women with a normal BMI $\left(\geq 18.5\right.$ and $\left.<25 \mathrm{~kg} / \mathrm{m}^{2}\right)$. Vigorous activity of $\geq 3$ hours per week (IRR of $4.50 ; 95 \% \mathrm{Cl} 1.72$ to 11.79 ) and a family history of stroke (IRR of $3.81 ; 95 \% \mathrm{Cl} 1.31$ to 11.07) were more strongly related to stillbirth risk among women with a normal BMI than that observed for all women combined. Established risk factors also associated with stillbirth risk.

Conclusions The study identified physical activity and family history of stroke as potential new risk factors for stillbirth delivery.

\section{INTRODUCTION}

Stillbirth affects 2.6million births each year globally ${ }^{1}$ and approximately 4 in 1000

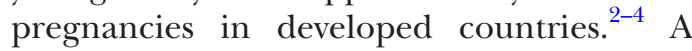
systematic review of 96 population-based studies from high-income countries identified
Strengths and limitations of this study

- Prospective cohort study design assessing behaviours prior to pregnancy with successful linkages to the Medical Birth Registry of Norway for ascertainment of stillbirth deliveries.

- Physical activity questions used in the survey had been validated using metabolic equivalent of tasks min per week scores obtained from the long-form of the International Physical Activity Questionnaire.

- The small sample size of stillbirths is a limitation where stillbirth rates within stratum of risk factors may be unstable and lead to biased results.

the following major risk factors: fetal growth restriction, placental abruption, maternal age over 35 years., overweight/obesity, maternal smoking, pre-existing diabetes and hypertension and primiparity. ${ }^{5}$ Also, stillbirth in a prior pregnancy conveys risk of recurrence in future pregnancies. ${ }^{6}$ A sizeable proportion of stillbirths are unexplained deaths given varying and suboptimal classification systems where important information is lost. ${ }^{7}$ Fetal growth restriction, however, is now recognised as the major underlying cause of stillbirth with an estimated population attributable fraction of $37.4 \%$ observed in England. ${ }^{4}$

In addition to being a traumatic loss for parents, there is evidence of an increased risk for adverse health outcomes for women who have had a fetal loss. In a Dutch case-control study of ischaemic stroke and myocardial infarction among women who had at least one pregnancy, women who had a stillbirth delivery had an increased risk of arterial thrombosis (OR 2.14; 95\% CI 1.15 to 4.00 ) and ischaemic stroke (OR 2.54; 95\% CI 1.12 to 5.72) compared with women without any pregnancy loss in analyses adjusted for age, region and year of indexed event. ${ }^{8}$ In a study from Norway, mothers with perinatal losses had an increased risk of cardiovascular 
mortality (HR $1.8 ; 95 \%$ CI 1.5 to 2.1 ). ${ }^{9}$ In the EPIC Heidelberg study of over 11000 women (33-66 years of age), those who experienced a stillbirth had a threefold increased risk of myocardial infarction (HR 3.4; 95\% CI 1.53 to 7.70 ) compared with women with no history of stillbirth, while findings were weaker for stroke (HR 1.8; $95 \%$ CI 0.65 to 5.05$).{ }^{10}$ Also, in a recent meta-analyses, a pooled relative risk for stroke of 1.86 (95\% CI, 1.15 to 3.02) was identified in women with a history of stillbirth delivery compared with women who had pregnancies without complications. ${ }^{11}$

Because the influence of pregravid factors on the risk of stillbirth is not known, our study objectives were to evaluate pregravid lifestyle and conventional cardiovascular disease risk factors for their association with subsequent stillbirth delivery in a cohort of female health survey participants followed via linkages to the Medical Birth Registry of Norway.

\section{METHODS}

Cohort Norway (CONOR) health survey participants were assessed between 1994 and 2003 and were linked, using a personal identification number, to the Medical Birth Registry of Norway for births following CONOR participation (through to 31 December 2012). Details of the study population are provided elsewhere. ${ }^{12} 13$ The CONOR surveys that included women of reproductive age were HUBRO Study, Oslo (24.2\%), the Nord-Trøndelag Health Study (HUNT) (49.9\%) and Tromsø Study $(21.3 \%)$. The majority of CONOR participants were ethnic Norwegians, but only $61.9 \%$ of women of reproductive age in Oslo surveys were born in Norway, given an immigrant component in Oslo.

Exclusions for the current paper include: multiple birth pregnancies, non-viable deliveries under 22 weeks gestation and mother pregnant during or delivered $<1$ year prior to CONOR participation.

\section{COHORT NORWAY AND MEDICAL BIRTH REGISTRY OF NORWAY ASSESSMENTS}

Assessments in CONOR included height and weight, non-fasting lipids, diabetes, history of antihypertensive medication usage and a family history of diabetes, stroke or myocardial infarction prior to age 60 years in first degree relatives. ${ }^{12}$ Past-year weekly leisure-time light and vigorous physical activity were also assessed. ${ }^{14}$ Vigorous activity was defined by activity resulting in sweating and/or shortness of breath and was categorised as $<1$ hour, $1-2$ hours and $\geq 3$ hours per week. Light activity (not resulting in sweating or shortness of breath) was categorised into $<3$ hours and $\geq 3$ hours per week. In a validation substudy, the CONOR physical activity questions correlated with metabolic equivalent of tasks (METs min per week) obtained from the long-form of the International Physical Activity Questionnaire. ${ }^{15}$ In the validation study, those that reported vigorous physical activity of 3 hours per week or more were 2.5 times more active compared with those engaging in vigorous physical activity $<1$ hour per week (ie, vigorous activity of 5020 METs min/week versus slightly less than 2020 METs min/week). Similarly, those engaging in light physical activity of $\geq 3$ hours per week were 1.5 times more active compared with those reporting $<3$ hours of light physical activity per week. ${ }^{14}$

CONOR assessments also included blood pressure (BP) where the mean of the last two of three systolic and diastolic BP readings taken by an automatic device (DINAMAP, Criticon, Tampa, Florida, USA) was used in analyses of diastolic and systolic BP quartiles.

The Medical Birth Registry ascertained stillbirth deliveries occurring at 22 weeks gestation or greater. Pregnancy characteristics have extensively been studied for their association with stillbirth delivery and were evaluated to determine consistencies between our cohort with that of the prevailing literature. In brief, we evaluated prior fetal losses, gestational hypertension diagnosis (systolic BP $\geq 140 \mathrm{~mm} \mathrm{Hg}$ and/or a diastolic BP $\geq 90 \mathrm{~mm} \mathrm{Hg}$ after 20 weeks of gestation), pre-eclampsia (gestational hypertension plus proteinuria: $>0.3 \mathrm{~g}$ in 24 hours urine or $\geq 1$ point increase on a urinary dipstick), ${ }^{16}$ and small for gestational age (SGA) using sex-specific birthweight z-scores. ${ }^{17}$

Missing data were low $(<0.1 \%)$ for the majority of CONOR survey parameters evaluated with the exception of baseline smoking (5\%) and physical activity (7\%).

\section{STATISTICAL METHODS}

Mothers' baseline characteristics as mean (SD) or per cent by stillbirth and live birth status were evaluated for statistically significance difference using linear and Poisson regression for continuous and dichotomous characteristics respectively, where mother's pseudo-ID was used to account for clustering of women who contributed more than one pregnancy over the follow-up time period. The cluster variable adjusts the SE for the intraclass correlation of pregnancies within each mother. Further, Poisson regression provided incidence rate ratios (IRRs) and 95\% CI of characteristics for their prediction of stillbirth deliveries in unadjusted and adjusted models. Postestimation goodness of fit tests evaluated model fit. Multivariable adjusted analyses included age at baseline (years) and length of follow-up. For lipid variables, the multivariable model also included use of oral contraceptives at baseline. The median time since last meal was 2 hours (IQR 1-3): adjustment for this variable did not alter the lipid results.

\section{Additional analyses}

We also conducted a sensitivity analyses restricted to normal weight women (BMI $\geq 18.5$ and $<25.0 \mathrm{~kg} / \mathrm{m}^{2}$ ) given that risk factors tend to cluster with higher BMI and because of the potential for bias where overweight/obese women may experience being out of breath or sweating at lower levels of physical exertion than women with a normal BMI. Further, we also re-evaluated significant 
findings in analyses adding congenital malformations noted at birth as a covariate.

Stata 14 was used in all analyses; statistical significance was determined by $\mathrm{p}<0.05$.

\section{RESULTS}

Record linkages identified 17320 births with a mother who participated in CONOR prior to delivery. After exclusions, the sample size for analyses was 13497 singleton births to 8478 women, representing 1.59 births/woman. At the CONOR baseline survey, the mean (SD) age was 27.9 (4.5) years and the mean BMI was $23.9(3.8) \mathrm{kg} /$ $\mathrm{m}^{2}, 26.9 \%$ smoked daily, $48.1 \%$ engaged in past-year light physical activity of $\geq 3$ hours/week, while $15.8 \%$ reported engaging in vigorous physical activity of $\geq 3$ hours/week. At the time of delivery, the mean (SD) maternal age was 33.0 years (4.4) with a mean (SD) length of follow-up from the CONOR baseline survey of 5.5 years (3.5) (min$\max 0.5-17.5)$.

There were 54 fetal losses among the 13497 deliveries (4 per 1000): 52 of whom had a registered birth weight providing a median birth weight of $930 \mathrm{~g}$ (IQR 520-2676). Birth weight for gestational age z-scores were significantly lower among stillbirths (median of -1.38 ; IQR -2.22 , -0.01 ) than among livebirths (median 0.08; IQR -0.57 , $0.76, \mathrm{p}<0.0001)$. Likewise, the median gestational age was 31 weeks (IQR 24, 36.5) for stillbirth deliveries versus 40 weeks (IQR 39, 41) for live births $(\mathrm{p}<0.0001)$. There were 541 births that had a congenital malformation $(4 \%$ of 13497 births), of whom four were stillbirth deliveries $(7.4 \%)$.

In the unadjusted comparisons of baseline characteristics between stillbirths and livebirths, the mean BMI and the per cent reporting 3 hours or more of past-year vigorous physical activity was significantly higher while the per cent reporting 3 hours a week of light physical activity was lower in mothers of stillbirths compared with mothers of live births (table 1).

In analyses adjusting for baseline age and length of follow-up, the risk of stillbirth delivery was greater among women who at baseline were over 35 years of age, obese and among those who reported a family history of stroke (table 2). Further, baseline past-year light physical activity of 3 hours or more per week associated with lower risk (IRR of $0.53 ; 95 \%$ CI 0.32 to 0.93 ), while vigorous physical activity of $\geq 3$ hours per week associated with an increased risk of stillbirth delivery (IRR 2.46; 95\% CI 1.23 to 4.90). Light and vigorous physical activity were not mutually exclusive as some women engaged in both, none or in predominately light or vigorous activity. There was no evidence of effect modification between the two forms of activity. The results were stronger when both forms of physical activity were entered into the model together (ie, IRR for light physical activity became $0.40,95 \%$ CI 0.21 to 0.73 and the IRR for $\geq 3$ hours of vigorous activity became $3.99,95 \%$ CI 1.95 to 8.19 ) adjusting for baseline age and length of follow-up.

The highest quartile of baseline diastolic BP $(>73.5 \mathrm{~mm}$ $\mathrm{Hg}$ ) did not convey a statistically significant association with increased risk of stillbirth (IRR 2.02; 95\% CI 0.94 to $4.31, \mathrm{p}=0.07)$. A high ratio of total cholesterol/HDL cholesterol, an elevated triglyceride value, quartiles of triglycerides, total and HDL cholesterol and a high triglyceride/HDL cholesterol ratio ( $\geq 90$ th percentile) were not associated with risk of stillbirth delivery (data not shown). A family history of myocardial infarction

Table 1 Baseline descriptive characteristics by subsequent stillbirth among singleton deliveries: Cohort Norway (CONOR) and Medical Birth Registry of Norway

\begin{tabular}{lll}
\hline Baseline characteristics & $\begin{array}{l}\text { Stillbirth }(\mathbf{n}=\mathbf{5 4}) \\
\text { Mean (SD) }\end{array}$ & $\begin{array}{l}\text { Livebirths }(\mathbf{n}=\mathbf{1 3 4 4 3}) \\
\text { Mean (SD) }\end{array}$ \\
\hline Age (years) & $33.6(4.36)$ & $32.9(4.4)$ \\
Body mass index $\left(\mathrm{kg} / \mathrm{m}^{2}\right)$ & $25.6(4.9)$ & $23.9(3.8)^{*}$ \\
\hline Light physical activity 3 hours or more per week (\%) & 33.3 & $48.7^{\star}$ \\
Vigorous physical activity 3 hours or more per week (\%) & 27.5 & $16.8^{\star}$ \\
Weekly alcohol consumption (\%) & 18.5 & 22.2 \\
\hline Daily smoking (\%) & 26.9 & 27.1 \\
\hline Mean systolic BP (mm Hg) & $120.2(11.9)$ & $119.1(11.04)$ \\
\hline Mean diastolic BP (mm Hg) & $71.9(9.84)$ & $69.0(8.5)$ \\
\hline Total cholesterol (mmol/L) & $4.91(0.94)$ & $4.90(0.92)$ \\
\hline HDL-cholesterol (mmol/L) & $1.54(0.49)$ & $1.54(0.36)$ \\
Triglycerides (mmol/L) & $1.06(0.49)$ & $1.15(0.69)$ \\
Any college or university education (\%) & 24.1 & 24.0 \\
\hline
\end{tabular}

*13 497 births to 8478 women, representing 1.59 births/woman; 54 stillbirths represent 54 unique women. Exclusions: births <22 weeks, and mother pregnant during or delivered $<1$ year prior to CONOR participation.

$\mathrm{p}<0.05$, unadjusted linear and Poisson regression for group differences in means and per cent, entering mother's pseudo-ID as cluster variable. 


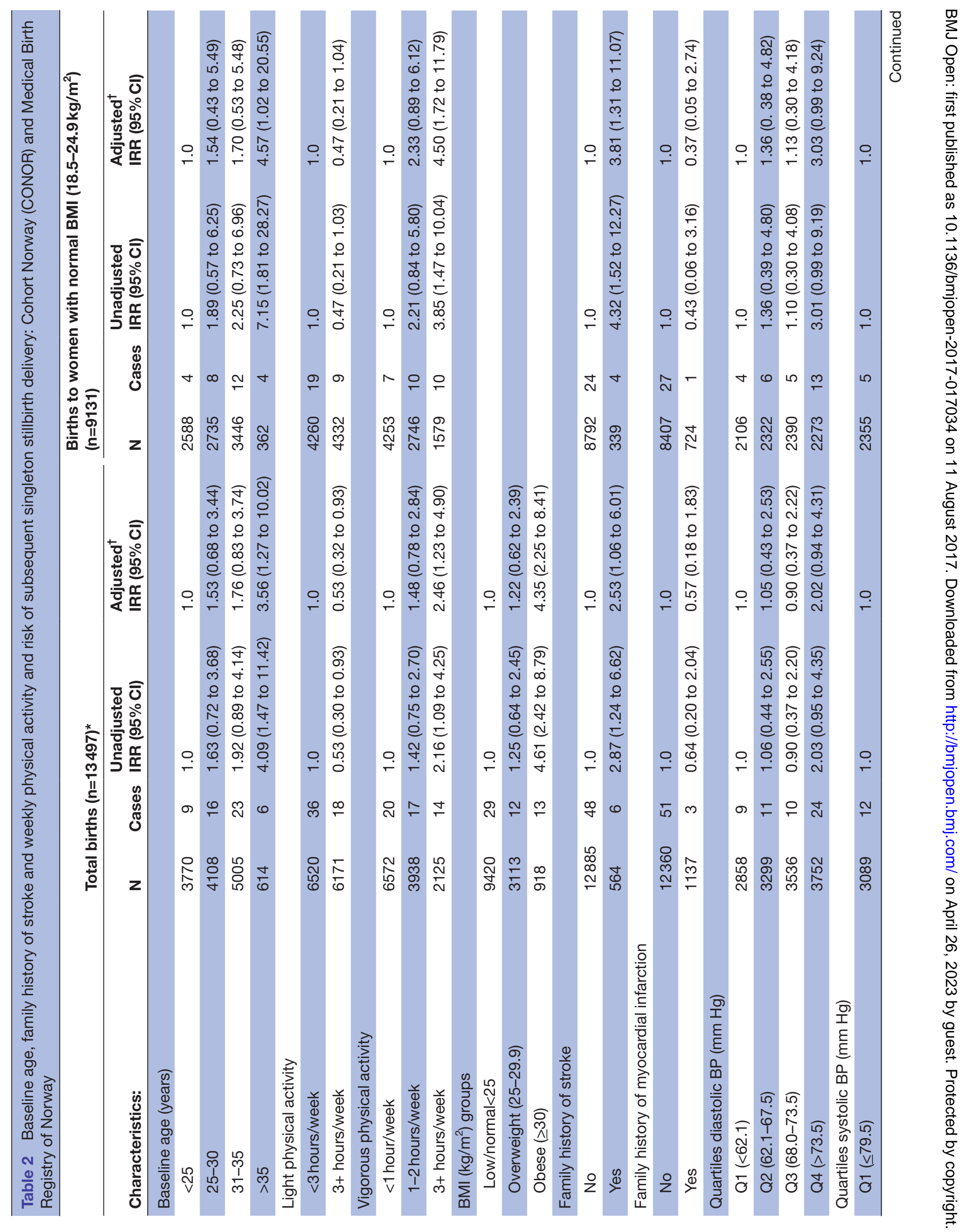




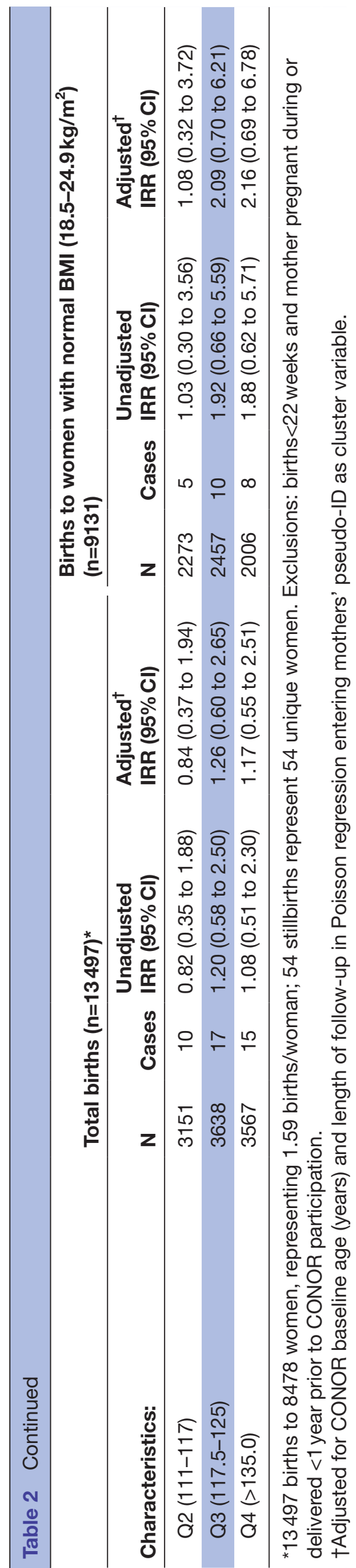

prior to age 60 (table 2), a family history of diabetes, and region of survey did not relate to risk of stillbirth (data not shown).

Hypertension during pregnancy, a delivery with a small weight for gestational age, a prior fetal loss and a prior history of gestational hypertension were associated with increased risk for stillbirth as expected (data not shown).

\section{ADDITIONAL ANALYSES}

In the sensitivity analyses limited to 9131 births to women with a normal BMI $\left(\geq 18.5\right.$ and $\left.<25 \mathrm{~kg} / \mathrm{m}^{2}\right)$, a baseline report of past-year vigorous activity of $\geq 3$ hours per week had a stronger association with stillbirth risk (IRR of 4.50; 95\% CI 1.72 to 11.79 ) than that observed for births to all women combined. Further, a family history of stroke (IRR 3.81; 95\% CI 1.31 to 11.07) and a diastolic BP in the highest quartile (IRR 3.03; 95\% CI 0.99 to $9.24 ; \mathrm{p}=0.06$ ) were more strongly related to stillbirth risk among women with a normal BMI than in the analyses of all women combined adjusting for baseline age and length of follow-up (table 2).

In analyses adding congenital malformation as a covariate, the protective association of light physical activity and the deleterious association of vigorous physical activity and a family history of stroke remained significant for all births and for births to women with a normal BMI.

\section{Supplemental post hoc analyses related to physical activity}

Post hoc analyses evaluated potential underlying issues that could help explain the physical activity results observed. Statistically significantly but modestly lower prepregnancy BMI was observed among those reporting light physical activity of $\geq 3$ hours per week versus less (Beta -0.45 (SE 0.07), p<0.001), and among those reporting weekly vigorous activity of $1-2$ hours (Beta -0.25 (SE 0.08), $\mathrm{p}<0.001$ ), and $\geq 3$ hours (Beta -0.57 (SE 0.09), $\mathrm{p}<0.001)$ relative to $<1$ hour of weekly vigorous activity in age-adjusted linear regression analyses. We found no evidence that vigorously active women with a stillbirth were more obese. The unadjusted mean prepregnancy BMI of vigorous physically active women (3+ hours/ week) who had a stillbirth $\left(23.6 \mathrm{~kg} / \mathrm{m}^{2}, 95 \%\right.$ CI 21.8 to 25.4) was similar to vigorously active women with a live birth $\left(23.4 \mathrm{~kg} / \mathrm{m}^{2}, 95 \%\right.$ CI 23.2 to 23.6$)$ and lower than less active women with a stillbirth $\left(26.1 \mathrm{~kg} / \mathrm{m}^{2}, 95 \%\right.$ CI 24.5 to 27.7). Similar birthweight z-scores and gestational ages were observed for stillbirths regardless of the physical activity level of mothers.

\section{DISCUSSION}

The study identified established risk factors for stillbirths in addition to novel findings regarding a family history of stroke and women's physical activity as associates of stillbirth risk. In terms of modifiable risk factors, vigorous physical activity of $\geq 3$ hours per week associated with 
increased risk while light physical activity of $\geq 3$ hours per week associated with reduced risk of stillbirth delivery relative to their respective less active comparison groups. In our previous evaluation of the preconception risk factors for pre-eclampsia in the same cohort, light and vigorous activity shared a similar protective direction in their association with subsequent risk of pre-eclampsia and were, therefore, combined into one activity category. ${ }^{12}$ We are not aware of other studies that have evaluated preconception physical activity and stillbirth risk. Any type of physical activity prior to and during pregnancy would likely result in greater weight management and, given the striking association of BMI with stillbirth risk, greater weight management would be a means of protection. There are, however, theoretical considerations and empirical evidence for a potentially beneficial effect of light and a detrimental effect of vigorous physical activity during pregnancy on risk of fetal losses.

Protective mechanisms of physical activity include reduced oxidative stress and inflammation, ${ }^{18}$ improved endothelial function, placental growth and vascular development. ${ }^{19} 20$ There are complex exercise-induced stimuli and response mechanisms where a periodic low placental perfusion, resulting from redirection of blood to mother's working muscles, would stimulate angiogenesis and placental growth. ${ }^{19}{ }^{20}$ In a review of animal and human literature regarding weight-bearing exercise during pregnancy, beneficial increases in placental function and resting maternal plasma volume, intervillous space blood volume and cardiac output were identified. ${ }^{19}$ In a review of the existing evidence by the Expert Committee on Exercise and Pregnancy of the International Olympic Committee, light-to-moderate physical activity was not deemed to increase the risk of miscarriage (defined as a fetal loss $<22$ weeks gestation). ${ }^{21}$ Our results contribute meaningfully to the literature with evidence that women routinely engaging in light physical activity prior to pregnancy had reduced risk of stillbirth in subsequent pregnancies.

High-intensity exercise, on the other hand, may pose risk, but the available literature is sparse and the level of evidence was considered low-to-moderate by the IOC Expert Committee. ${ }^{21}$ High-intensity exercise has been observed to result in a high umbilical artery pulsatility index and in fetal bradycardia during treadmill monitoring of six elite athletes when women exercised above $90 \%$ of their maximum maternal heart rate, ${ }^{22}$ results that corroborate earlier findings of fetal bradycardia observed in treadmill tests in 3 out of 19 healthy active women. ${ }^{23}$ Also, in a small study of 162 women planning pregnancy and keeping a $\log$ of their physical activity overtime, miscarriage was associated with exercise intensity around the time of implantation. ${ }^{24}$ Early fetal losses were evaluated in a large substudy of the Danish National Birth Cohort, involving telephone interviewing of women during pregnancy in which a subsample of the interviews occurred after a miscarriage. They reported a dose-response between the amount of time engaged in weekly physical activity and miscarriage risk. ${ }^{25}$ High-impact exercise, in particular, was associated with a notable increased risk of miscarriage at $<11$ weeks gestation, 11-14 weeks and 15-18 weeks gestation (HR of 3.6: 95\% CI 2.5 to 5.2; HR of 4.2; $95 \%$ CI 3.4 to 5.2; and HR of $2.1 ; 95 \%$ CI of 1.2 to 3.5$)$, respectively, but no excess risk was observed at 19-22 weeks gestation. In the same study, low impact and workout/fitness training was also associated with increased risk of miscarriage but to a lesser degree than that observed for high-impact exercise. In the Norwegian Mother and Child Cohort, physically active obese women did not have statistically significantly higher risk of perinatal death compared with non-active obese women. ${ }^{26}$ However, the study did not separately evaluate exercise intensity. In a more recent Danish study evaluating late miscarriages and stillbirths, no significant association was observed between physical activity which was simply dichotomised as yes versus no to the question, "Do you get any kind of exercise during pregnancy?". ${ }^{27}$ In an older study of over 20000 US women followed from early pregnancy, no association was observed between non-validated questions regarding light, moderate and vigorous physical activity and fetal or neonatal deaths. ${ }^{28}$

The observation that a family history of stroke associated with risk of stillbirth is interesting given the recent meta-analyses of the literature indicating a greater risk of stroke in women who had experienced pregnancy losses. ${ }^{11}$ Inherited thrombophilia has been related to both stillbirth risk ${ }^{29}{ }^{30}$ and ischaemic stroke, ${ }^{31}$ but studies are conflicting. ${ }^{32-34}$ Also, in 40-year-old Swedish women, a history of stillbirth delivery was associated with higher systolic BP, but not significantly with diastolic BP in multivariable analyses. ${ }^{35}$ In contrast, we found no evidence of an association between baseline systolic BP and later stillbirth risk, but did find nearly significantly higher risk for stillbirth associated with the top quartile of baseline diastolic BP. Further research exploring familial history of disease, inherited traits and systolic versus diastolic BP trajectories with risk of stillbirth and other fetal losses would be worthwhile.

While the current study also identified as significant many of the known risk factors for stillbirth delivery, baseline smoking did not relate to stillbirth in the current study, contrary to expectations. ${ }^{36}$ We assume that a large per cent of smokers in the baseline CONOR survey had quit within the follow-up period given the striking declines in smoking prevalence noted in Norway during this past decade. ${ }^{37}$ Among the 3484 baseline CONOR smokers, there was pregnancy smoking information on $2204(63.3 \%)$ of whom $55 \%$ indicated no smoking during pregnancy (ie, likely had quit smoking during the follow-up period).

Strengths of the current study involve the prospective study design following women of reproductive age who participated in the CONOR health surveys with linkages to the Medical Birth Registry of Norway for ascertainment of all subsequent births. Another strength is that the CONOR physical activity questions correlated with METs 
min per week obtained from the validated long-form of the International Physical Activity Questionnaire and, although weakly, with greater cardiorespiratory fitness $\mathrm{VO}_{2 \max }$ treadmill test, and improved BMI, waist-hip ratio and non-fasting lipids in women. ${ }^{13}$ Further evidence of construct validity of the physical activity questionnaires is that weekly baseline light and vigorous activity significantly associated with lower baseline BMI in age-adjusted linear regression analyses.

Limitations include the low number of stillbirths which when coupled with an uncommon risk factor provided inadequate power to observe significant results. Further, given the small number of stillbirths, rates by stratum-specific risk factors could be unstable and potentially lead to biased associations. Therefore, corroboration of results by future research is desirable. Another limitation is that we do not have information regarding family history of hypertension and do not know the extent to which physical activity behaviours at the baseline survey related to behaviours during the conception and through pregnancy. Further, we cannot specify the intensity, duration, and frequency of vigorous activity through the questions administered. In addition, with a mean follow-up time of 5.5 years between CONOR participation and delivery, we cannot rule out that some of the women may have changed their lifestyle and thus their level of physical activity before pregnancy. Another limitation is that we had inadequate numbers to stratify analyses by early versus late stillbirths. Finally, because of the uniqueness of the data, we chose an exploratory design with no predefined hypotheses specifically regarding physical activity. These limitations highlight the need for future corroboration of the research findings.

\section{SUMMARY}

The study provides evidence that routine light physical activity could potentially reduce risk of stillbirth delivery: an important observation that extends the list of known or suspected benefits of physical activity in pregnancy. Further, the study adds to the evidence available that vigorous physical activity may pose harm to the fetus at least in terms of a potential increased risk of stillbirth. Because of the importance of physical activity in promoting health among women of reproductive age and in promoting healthy pregnancies, further studies are needed to identify safety thresholds for vigorous activity in pregnancy. Finally, given the small numbers with a family history of stroke, corroboration of findings and explorations of mechanisms by which a family history of stroke may convey greater risk of stillbirth require further study.

Acknowledgements We acknowledge the CONOR study participants and the CONOR Steering Committee and research teams that contributed the majority of participants for analyses: The Troms $\emptyset$ Study (IV and V), Troms and Finnmark Health Study (TROFINN), Oslo Health and Immigrant Health Study (HUBRO \& I-HUBRO) and HUNT II Survey, the HUNT. We also thank Hordaland Health Study (HUSK) and Oppland and Hedmark Health Study (OPPHED).
Contributors GME designed the secondary use of the data, conducted analyses, drafted original and revised text; GST and ØN planned and implemented the baseline CONOR health surveys; KK implemented quality assurance for the MBRN; $\emptyset \mathrm{N}$ and Jon M.G. Wickmann implemented data linkages. GST, ØN, Jl, and KK provided critical feedback and intellectual content. GME is guarantor.

Funding Secondary data analyses and record linkages were funded by the Norwegian Institute of Public Health and The Norwegian Sudden Infant Death and Stillbirth Society.

Disclaimer The funders did not influence the conduct of the study, analysis or interpretation of the data, the writing of this report or the decision to publish.

Competing interests None declared.

Patient consent Detail has been removed from this case description/these case descriptions to ensure anonymity. The editors and reviewers have seen the detailed information available and are satisfied that the information backs up the case the authors are making.

Ethics approval Regional Ethics Committee.

Provenance and peer review Not commissioned; externally peer reviewed.

Data sharing statement Bona fide researchers can apply to use data through formal protocols, review, and agreements (www.fhi.no).

Open Access This is an Open Access article distributed in accordance with the Creative Commons Attribution Non Commercial (CC BY-NC 4.0) license, which permits others to distribute, remix, adapt, build upon this work non-commercially, and license their derivative works on different terms, provided the original work is properly cited and the use is non-commercial. See: http://creativecommons.org/ licenses/by-nc/4.0/

(c) Article author(s) (or their employer(s) unless otherwise stated in the text of the article) 2017. All rights reserved. No commercial use is permitted unless otherwise expressly granted.

\section{REFERENCES}

1. Lawn JE, Blencowe $H$, Waiswa $P$, et al. Stillbirths: rates, risk factors, and acceleration towards 2030. Lancet 2016;387:587-603.

2. Flenady V, Middleton P, Smith GC, et al. Stillbirths: the way forward in high-income countries. Lancet 2011;377:1703-17.

3. Man J, Hutchinson JC, Ashworth M, et al. Stillbirth and intrauterine fetal death: contemporary demographic features of $>1000$ cases from an urban population. Ultrasound Obstet Gynecol 2016;48:591-5.

4. Gardosi J, Madurasinghe V, Williams M, et al. Maternal and fetal risk factors for stillbirth: population based study. BMJ 2013;346:f108.

5. Flenady V, Koopmans L, Middleton P, et al. Major risk factors for stillbirth in high-income countries: a systematic review and metaanalysis. Lancet 2011;377:1331-40.

6. Melve KK, Skjaerven R, Rasmussen S, et al. Recurrence of stillbirth in sibships: population-based cohort study. Am J Epidemiol 2010;172:1123-30.

7. Flenady V, Frøen JF, Pinar $\mathrm{H}$, et al. An evaluation of classification systems for stillbirth. BMC Pregnancy Childbirth 2009;9:24.

8. Maino A, Siegerink B, Algra A, et al. Pregnancy loss and risk of ischaemic stroke and myocardial infarction. Br J Haematol 2016;174:302-9.

9. Halland F, Morken NH, DeRoo LA, et al. Long-term mortality in mothers with perinatal losses and risk modification by surviving children and attained education: a population-based cohort study. BMJ Open 2016;6:e012894.

10. Kharazmi E, Dossus L, Rohrmann S, et al. Pregnancy loss and risk of cardiovascular disease: a prospective population-based cohort study (EPIC-Heidelberg). Heart 2011;97:49-54.

11. Poorthuis $\mathrm{MH}$, Algra AM, Algra A, et al. Female- and Male-Specific risk factors for Stroke: a systematic review and Meta-analysis. JAMA Neurol 2017;74:75-81.

12. Egeland GM, Klungsøyr K, Øyen N, et al. Preconception Cardiovascular risk factor differences between gestational hypertension and preeclampsia: cohort Norway Study. Hypertension 2016;67:1173-80.

13. Naess O, Søgaard AJ, Arnesen E, et al. Cohort profile: cohort of Norway (CONOR). Int J Epidemiol 2008;37:481-5.

14. Graff-Iversen S, Anderssen SA, Holme IM, et al. Two short questionnaires on leisure-time physical activity compared with serum lipids, anthropometric measurements and aerobic power 
in a suburban population from Oslo, Norway. Eur J Epidemiol 2008:23:167-74.

15. Craig CL, Marshall AL, Sjöström M, et al. International physical activity questionnaire: 12 -country reliability and validity. Med Sci Sports Exerc 2003;35:1381-95.

16. Klungsøyr K, Harmon QE, Skard LB, et al. Validity of pre-eclampsia registration in the medical birth registry of Norway for women participating in the norwegian mother and child cohort study, 19992010. Paediatr Perinat Epidemiol 2014;28:362-71.

17. Skjaerven R, Gjessing HK, Bakketeig LS. Birthweight by gestational age in Norway. Acta Obstet Gynecol Scand 2000;79:440-9.

18. Kasapis $C$, Thompson PD. The effects of physical activity on serum C-reactive protein and inflammatory markers: a systematic review. $J$ Am Coll Cardiol 2005;45:1563-9.

19. Clapp JF. The effects of maternal exercise on fetal oxygenation and feto-placental growth. Eur J Obstet Gynecol Reprod Biol 2003;110 Suppl 1(suppl 1):S80-S85.

20. Genest DS, Falcao S, Gutkowska J, et al. Impact of exercise training on preeclampsia: potential preventive mechanisms. Hypertension 2012;60:1104-9.

21. Bø $\mathrm{K}$, Artal $\mathrm{R}$, Barakat $\mathrm{R}$, et al. Exercise and pregnancy in recreational and elite Athletes: 2016 evidence summary from the IOC expert group meeting, Lausanne. Part 2-the effect of exercise on the fetus, labour and birth. Br J Sports Med 2016;50:1297-305.

22. Salvesen $K A ̊$, Hem E, Sundgot-Borgen J. Fetal wellbeing may be compromised during strenuous exercise among pregnant elite Athletes. Br J Sports Med 2012;46:279-83.

23. Artal R, Romem $\mathrm{Y}$, Paul $\mathrm{RH}$, et al. Fetal bradycardia induced by maternal exercise. Lancet 1984;2:258-60.

24. Hjollund NH, Jensen TK, Bonde JP, et al. Spontaneous abortion and physical strain around implantation: a follow-up study of firstpregnancy planners. Epidemiology 2000;11:18-23.

25. Madsen M, Jørgensen T, Jensen ML, et al. Leisure time physical exercise during pregnancy and the risk of miscarriage: a study within the danish National Birth Cohort. BJOG 2007;114:1419-26.

26. Sorbye LM, Klungsoyr K, Samdal O, et al. Pre-pregnant body mass index and recreational physical activity: effects on perinatal mortality in a prospective pregnancy cohort. BJOG 2015;122:1322-30.
27. Morales-Suárez-Varela M, Nohr EA, Bech BH, et al. Smoking, physical exercise, BMl and late foetal death: a study within the danish National Birth Cohort. Eur J Epidemiol 2016;31:999-1009.

28. Rose NC, Haddow JE, Palomaki GE, et al. Self-rated physical activity level during the second trimester and pregnancy outcome. Obstet Gynecol 1991;78:1078-80.

29. Nurk E, Tell GS, Refsum H, et al. Factor V Leiden, pregnancy complications and adverse outcomes: the Hordaland Homocysteine Study. QJM 2006;99:289-98.

30. Helgadottir LB, Skjeldestad FE, Jacobsen AF, et al. The association of inherited thrombophilia and intrauterine fetal death: a case-control study. Blood Coagul Fibrinolysis 2011;22:651-6.

31. Tasdemir S, Erdem HB, Sahin I, et al. Correlation with platelet parameters and genetic markers of Thrombophilia Panel (Factor II g.20210G>A, factor V leiden, MTHFR (C677T, A1298C), PAI-1, $\beta$-Fibrinogen, factor XIIIA (V34L), Glycoprotein Illa (L33P)) in ischemic strokes. Neuromolecular Med 2016;18:170-6.

32. Silver RM, Saade GR, Thorsten V, et al. Factor V Leiden, prothrombin G20210A, and methylene tetrahydrofolate reductase mutations and stillbirth: the Stillbirth Collaborative Research Network. Am J Obstet Gynecol 2016;215:468.e1-468.e17. e1-17.

33. Berge E, Haug KB, Sandset EC, et al. The factor V leiden, prothrombin gene $20210 \mathrm{ga}$, methylenetetrahydrofolate reductase $677 \mathrm{ct}$ and platelet glycoprotein IIla 1565tc mutations in patients with acute ischemic stroke and atrial fibrillation. Stroke 2007;38:1069-71.

34. Pahus SH, Hansen AT, Hvas AM. Thrombophilia testing in young patients with ischemic stroke. Thromb Res 2016;137:108-12.

35. Parikh NI, Norberg M, Ingelsson E, et al. Association of Pregnancy complications and characteristics with future risk of elevated blood pressure: the Västerbotten intervention program. Hypertension 2017;69:475-83.

36. Marufu TC, Ahankari A, Coleman T, et al. Maternal smoking and the risk of still birth: systematic review and meta-analysis. BMC Public Health 2015;15:239.

37. Grøtvedt L, Kvalvik LG, Grøholt EK, et al. Development of Social and Demographic differences in Maternal Smoking between 1999 and 2014 in Norway. Nicotine Tob Res 2017;19:539-46. 